\title{
Surface-Impedance Boundary Conditions in Time-Domain Finite-Element Calculations using the Magnetic-Vector-Potential Formulation
}

\author{
J. Gyselinck ${ }^{1}$, P. Dular ${ }^{2,3}$, C. Geuzaine ${ }^{2}$ and R. V. Sabariego ${ }^{2}$ \\ ${ }^{1}$ Dept. of Bio-, Electro- and Mechanical Systems (BEAMS), Université Libre de Bruxelles (ULB), Belgium \\ ${ }^{2}$ Dept. of Electrical Engineering and Computer Science, University of Liège, Belgium \\ ${ }^{3}$ Fonds de la Recherche Scientifique - FNRS, Belgium
}

\begin{abstract}
The authors propose a novel time-domain extension of the well-known frequency-domain surface-impedance method in computational magnetodynamics. Herein the 1-D eddy-current problem in a massive conducting region (semi-infinite slab) is considered via a number of exponentially decreasing trigonometric basis functions that cover the relevant skin-depth (or frequency) range of the application at hand. The method is elaborated for the 3-D magnetic-vector-potential formulation and is applied to a simple 2-D test case. Results are shown to converge well to those obtained with an accurate brute-force FE model.
\end{abstract}

Index Terms-Finite-element methods, surface-impedance boundary conditions, magnetodynamics, time-domain analysis

\section{INTRODUCTION}

$\mathbf{S}$ URFACE-IMPEDANCE boundary conditions (SIBCs) are widely applied in frequency-domain eddy-current problems for considering massive conducting regions. The approach is based on the relation between the tangential components of the electric and the magnetic field at the surface of the conducting region and allows to discretize the surface of the region only. A necessary condition is that at the considered frequency the skin depth is sufficiently small compared to the depth or curvature of the conducting region. Several refinements have been presented in literature, which mostly concern the curvature of the surface [1], but also the saturation of the material [2].

The few time-domain extensions proposed to date are mostly based on the fast Fourier transform [3], on the explicit and separate resolution of a large number of 1-D FE models (with classical nodal basis functions) parallel to the main 3-D FE model [4], or on the convolution product [1]. The time-domain approach presented in this paper relies on the spatial discretisation of the 1-D eddy-current problem by means of dedicated basis functions which are derived from the analytical frequency-domain solution. The method is first briefly elaborated in sections II and III and then validated by means of a simple 2-D test case in section IV.

\section{1-D EDDY CURRENT PROBLEM IN SEMI-INFINITE SLAB}

\section{A. Magnetic-vector-potential formulation}

In this paper we are concerned with low-frequency eddycurrent problems. The following Maxwell equations and constitutive laws are taken into account:

$$
\operatorname{div} \underline{b}=0, \quad \begin{gathered}
\operatorname{curl} \underline{h}=\underline{j}, \quad \operatorname{curl} \underline{e}=-\partial_{t} \underline{b}, \\
\underline{j}=\sigma \underline{e}, \quad \underline{b}=\mu \underline{h},
\end{gathered}
$$

where the vector fields $\underline{b}, \underline{h}, j$ and $\underline{e}$ are the flux density (or induction), the magnetic field, the current density and the electric field respectively; $\sigma, \mu$ and $\nu=1 / \mu$ are the conductivity, the permeability and the reluctivity respectively. We consider a bounded domain $\Omega$ in the 3-D Euclidean space

This work was partly supported by the Belgian Science Policy (IAP P6/21). that comprises linear and homogenous media only, i.e. $\sigma, \mu$ and $\nu$ are constant scalars for each medium present in $\Omega$.

We introduce the magnetic vector potential $\underline{a}$ in order to satisfy $(1 \mathrm{a}, \mathrm{c})$ strongly:

$$
\underline{b}=\operatorname{curl} \underline{a}, \quad \underline{e}=-\partial_{t} \underline{a} .
$$

The remaining equations in (1-2) lead to the following partial differential equation:

$$
\operatorname{curl}(\nu \operatorname{curl} \underline{a})=\underline{j}_{s}-\sigma \partial_{t} \underline{a},
$$

where $j_{s}$ is the prescribed source current density in a subdomain $\Omega_{s}$ of $\Omega$; in another conducting subdomain $\Omega_{c}$ the current density is induced and given by $-\sigma \partial_{t} \underline{a}$.

\section{B. Analytical solution for 1-D eddy-current model}

We consider now the 1-D eddy-current problem in a semiinfinite slab $(0 \leq x \leq \infty)$, with $\underline{b}(x, t)$ and $\underline{h}(x, t)$ parallel to the $z$-axis, and $j(x, t)$ and $\underline{e}(x, t)$ parallel to the $y$-axis. The source current density $j_{s}$ is zero. With the $y$-component of the vector potential $\underline{a}(x, t)$ denoted by $a(x, t)$, (4) becomes

$$
\partial_{x}^{2} a=\sigma \mu \partial_{t} a \quad \text { with } a(x=\infty, t)=0,
$$

where the boundary condition at infinity $(x=\infty)$ ensures the uniqueness of $a(x, t)$.

The sinusoidal steady-state solution at frequency $f$ (pulsation $\omega=2 \pi f)$, with boundary condition $a(x=0, t)=$ $\hat{a} \cos (\omega t+\phi)$, reads

$$
\begin{aligned}
a(x, t)= & \hat{a} e^{-x / \delta} \cos (x / \delta-\omega t-\phi), \\
= & \hat{a} \cos (\omega t+\phi) e^{-x / \delta} \cos (x / \delta) \\
& +\hat{a} \sin (\omega t+\phi) e^{-x / \delta} \sin (x / \delta),
\end{aligned}
$$

where $\delta=\sqrt{2 /(\mu \sigma \omega)}$ is the skin depth and $\phi$ an arbitrary phase angle.

Using the complex notation (symbols in bold, imaginary unit $\sqrt{-1}$ denoted by $i$ ), we rewrite (6) as follows

$$
\begin{gathered}
a(x, t)=\Re\left(\hat{a} e^{-\frac{1+i}{\delta} x} e^{i(\omega t+\phi)}\right), \\
\Rightarrow \quad \boldsymbol{a}(x)=\hat{a} e^{i \phi} e^{-\frac{1+i}{\delta} x},
\end{gathered}
$$

which further leads to a relation between the induction $\underline{b}=$ 
curl $\underline{a}$ and the electric field $\underline{e}=-\partial_{t} \underline{a}$ at the surface $x=0$ :

$$
\left.\partial_{x} \boldsymbol{a}\right|_{x=0}=-\frac{1+\boldsymbol{i}}{\delta} \boldsymbol{a}(x=0) .
$$

It is this equation that is at the heart of the classical frequencydomain SIBC approach.

\section{Low-order time-domain model of semi-infinite slab}

We now develop a low-order model of the 1-D eddy-current problem by adopting $n$ skin depths $\delta_{k}, 1 \leq k \leq n$, in agreement with the frequency spectrum of the magnetic fields in the problem at hand (see e.g. the application example hereafter and the one in section III). Based on the analytical solution (7), we define the following $2 n$ basis functions:

$$
\begin{aligned}
& \alpha_{c 1}(x)=e^{-x / \delta_{1}} \cos \left(x / \delta_{1}\right), \\
& \alpha_{c k}(x)=e^{-x / \delta_{k}} \cos \left(x / \delta_{k}\right)-\alpha_{c 1}(x), \quad 2 \leq k \leq n, \\
& \alpha_{s k}(x)=e^{-x / \delta_{k}} \sin \left(x / \delta_{k}\right), \quad 1 \leq k \leq n .
\end{aligned}
$$

Note that all basis functions vanish at the boundary $x=0$ except the first one, for which we have $\alpha_{c 1}(x=0)=1$.

The associated expansion of $a(x, t)$ is written in matrix form as follows:

$$
a(x, t)=[A(t)]^{T}[\alpha(x)],
$$

with the $2 n \times 1$ column matrices $[A(t)]$ and $[\alpha(x)]$ given by

$$
\begin{aligned}
& {[A(t)]=\left[\begin{array}{llllll}
a_{c 1}(t) & \ldots & a_{c n}(t) & a_{s 1}(t) & \ldots & a_{s n}(t)
\end{array}\right]^{T},} \\
& {[\alpha(x)]=\left[\begin{array}{llllll}
\alpha_{c 1}(x) & \ldots & \alpha_{c n}(x) & \alpha_{s 1}(x) & \ldots & \alpha_{s n}(x)
\end{array}\right]^{T} .}
\end{aligned}
$$

The classical variational $\mathrm{FE}$ approach leads to a system of $2 n$ first-order differential equations in terms of the elements of $[A(t)]$ :

$$
[S][A]+[M] \partial_{t}[A]=0,
$$

where the elements of $[S]$ and of $[M]$ are given by

$$
\begin{aligned}
S_{i j} & =\nu \int_{0}^{\infty} \partial_{x} \alpha_{i}(x) \partial_{x} \alpha_{j}(x) d x, \\
M_{i j} & =\sigma \int_{0}^{\infty} \alpha_{i}(x) \alpha_{j}(x) d x .
\end{aligned}
$$

An analytical expression of these elements can be straightforwardly derived given the following three equations:

$$
\begin{gathered}
\int_{0}^{\infty} e^{-x / \delta_{k}} e^{-x / \delta_{l}}\left[\begin{array}{c}
\cos \left(x / \delta_{k}\right) \cos \left(x / \delta_{l}\right) \\
\cos \left(x / \delta_{k}\right) \sin \left(x / \delta_{l}\right) \\
\sin \left(x / \delta_{k}\right) \sin \left(x / \delta_{l}\right)
\end{array}\right] d x \\
=\frac{\delta_{k} \delta_{l}}{2\left(\delta_{k}+\delta_{l}\right)\left(\delta_{k}^{2}+\delta_{l}^{2}\right)}\left[\begin{array}{c}
\delta_{k}^{2}+\delta_{l}^{2}+\delta_{k} \delta_{l} \\
\delta_{k}^{2} \\
\delta_{k} \delta_{l}
\end{array}\right] .
\end{gathered}
$$

The associated positive-definite quadratic forms are the instantaneous magnetic energy density $w(t)$ and the instantaneous eddy-current loss density $p(t)$ :

$$
w=[A]^{T}[S][A] \text { and } p=\partial_{t}[A]^{T}[M] \partial_{t}[A] .
$$

Further note that $[S]$ and $[M]$ can be normalized considering e.g. the first skin depth $\delta_{1}$ and associated pulsation $\omega_{1}=$ $2 \nu /\left(\sigma \delta_{1}^{2}\right)$ :

$$
\left[S^{*}\right]=\frac{\delta_{1}}{\nu}[S] \quad \text { and } \quad\left[M^{*}\right]=\frac{1}{\delta_{1} \sigma}[M],
$$

where the dimensionless matrices $\left[S^{*}\right]$ and $\left[M^{*}\right]$ depend only on the $n-1$ skin-depth ratios $\delta_{2} / \delta_{1}=\sqrt{\omega_{1} / \omega_{2}}$ to $\delta_{n} / \delta_{1}=$ $\sqrt{\omega_{1} / \omega_{n}}$. Equation (17) then becomes

$$
\left[S^{*}\right][A]+\frac{2}{\omega_{1}}\left[M^{*}\right] \partial_{t}[A]=0 .
$$

\section{Validation and application example of low-order model}

We now modify (17) in order to have the surface value of the magnetic vector potential, i.e. $a_{x=0}(t)=a_{c 1}(t)$, as source for the eddy-current problem. By removing $a_{c 1}(t)$ from $[A(t)]$ we obtain the column matrix of $2 n-1$ effective degrees of freedom, denoted by $\left[A^{\prime}(t)\right]$, and the associated system of $2 n-1$ differential equations reads

$$
\left[S^{\prime}\right]\left[A^{\prime}\right]+\left[M^{\prime}\right] \partial_{t}\left[A^{\prime}\right]=-\left([s] a_{x=0}+[m] \partial_{t} a_{x=0}\right),
$$

where the square matrices $\left[S^{\prime}\right]$ and $\left[M^{\prime}\right]$ and the column matrices $[s]$ and $[m]$ are straightforwardly obtained from $[S]$ and $[M]$.

Considering the sinusoidal regime at pulsation $\omega$, (24) is transformed into

$$
\left(\left[S^{\prime}\right]+\boldsymbol{i} \omega\left[M^{\prime}\right]\right)\left[\boldsymbol{A}^{\prime}\right]=-([s]+\boldsymbol{i} \omega[m]) \boldsymbol{a}_{x=0},
$$

and the derivative $\partial_{x} \boldsymbol{a}(x)$ at the surface $x=0$ can be written as follows:

$$
\left.\partial_{x} \boldsymbol{a}(x)\right|_{x=0}=-\frac{1}{\delta_{1}} \boldsymbol{a}_{x=0}+[v]^{T}\left[\boldsymbol{A}^{\prime}\right],
$$

where the first $n-1$ elements of the column matrix $[v]$ are given by $1 / \lambda_{1}-1 / \lambda_{2}$ to $1 / \lambda_{1}-1 / \lambda_{n}$, and the last $n$ elements by $1 / \lambda_{1}$ to $1 / \lambda_{n}$.

We thus find the approximation of (10) given the $n$ pairs of basis functions of the low-order model:

$$
\left.\partial_{x} \boldsymbol{a}\right|_{x=0}=-\frac{\boldsymbol{Z}_{n}}{\delta} \boldsymbol{a}(x=0),
$$

where $Z_{n}$ is a function of $\delta / \delta_{1}$ (or $\left.\omega / \omega_{1}\right)$ and of the $n-1$ skin-depth ratios $\delta_{2} / \delta_{1}$ to $\delta_{n} / \delta_{1}$ :

$$
\boldsymbol{Z}_{n}=\frac{\delta}{\delta_{1}}+\delta[v]^{T}\left(\left[S^{\prime}\right]+\boldsymbol{i} \omega\left[M^{\prime}\right]\right)^{-1}([s]+\boldsymbol{i} \omega[m]) .
$$

When the skin depth $\delta$ is equal to one of the $n$ preset skin depths, the low-order model gives the exact result, i.e. $\boldsymbol{Z}_{n}(\delta)=1+i$, as the analytical solution can be restituted by two of the $2 n$ basis functions. For intermediate values of $\delta$ and values outside the range covered by the $n$ preset skin depths, some error is inevitable.

We verify this considering $n$ subsequently equal to 1,2 and 3 , and with $\omega_{2} / \omega_{1}=3$ and $\omega_{3} / \omega_{1}=5$. Figure 1 shows the real and imaginary part of $\boldsymbol{Z}_{1}, \boldsymbol{Z}_{2}$ and $\boldsymbol{Z}_{3}$ with $\delta / \delta_{1}$ ranging from $1 / 4$ to 2 (or $\omega / \omega_{1}$ ranging from $1 / 4$ to 16 ). One clearly observes the convergence of $\boldsymbol{Z}_{n}$ towards the exact solution (real and imaginary part both equal to 1 ) with increasing $n$.

\section{FE IMPLEMENTATION}

We consider a 3-D domain $\Omega$ that comprises a source domain $\Omega_{s}$ (with given $\underline{j}_{s}$ ) and a conducting domain $\Omega_{c}$ with induced current $\left(j={ }_{-}^{-} \sigma \partial_{t} \underline{a}\right)$. The SIBC method will be applied to a massive subdomain $\Omega_{m}$ of $\Omega_{c}$. In the classical (frequency-domain) approach the weak form of the Ampère 

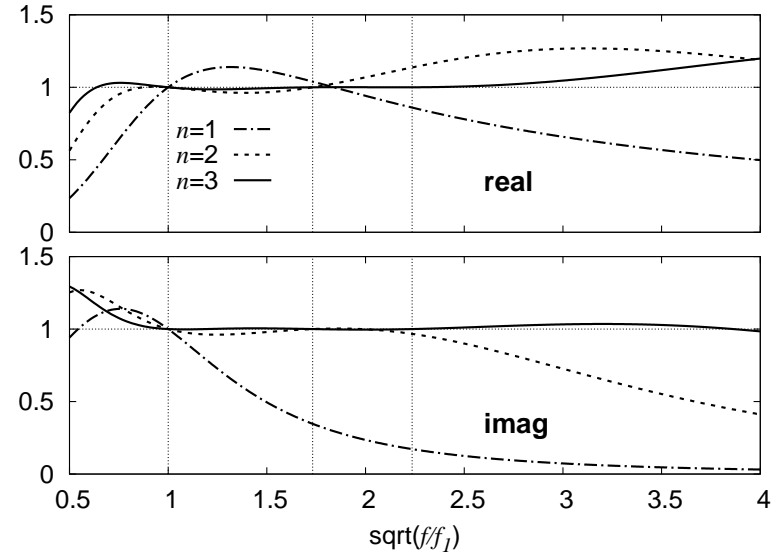

Fig. 1. Real and imaginary part of $\boldsymbol{Z}_{1}, \boldsymbol{Z}_{2}$ and $\boldsymbol{Z}_{3}$ as a function of $\sqrt{f / f_{1}}=$ $\sqrt{\omega / \omega_{1}}=\sqrt{\lambda_{1} / \lambda}$ with $\omega_{2} / \omega_{1}=3$ and $\omega_{3} / \omega_{1}=5$

law (4) is first written as follows:

$$
\begin{aligned}
\left(\nu \operatorname{curl} \underline{a}, \operatorname{curl} \underline{a}^{\prime}\right)_{\Omega / \Omega_{m}}+ & \left(\sigma \partial_{t} \underline{a}, \underline{a}^{\prime}\right)_{\Omega_{c} / \Omega_{m}}+ \\
& \left\langle\underline{n} \times \underline{h}, \underline{a}^{\prime}\right\rangle_{\partial \Omega_{m}}=\left(\underline{j}_{s}, \underline{a}^{\prime}\right)_{\Omega_{s}},
\end{aligned}
$$

where $(\cdot, \cdot)_{\Omega}$ and $\langle\cdot, \cdot\rangle_{\partial \Omega_{m}}$ are the integrals on the domain $\Omega$ and on the boundary $\partial \Omega_{m}$, respectively, of the product of the two arguments; $\underline{n}$ is the outward normal on $\partial \Omega_{m}$. The integral of the tangential magnetic field $\underline{n} \times \underline{h}$ on $\partial \Omega_{m}$ is then expressed in terms of the tangential electric field $\underline{n} \times \underline{e}=-\underline{n} \times \partial_{t} \underline{a}$ thanks to (10). The domain $\Omega / \Omega_{m}$, its boundary and the weak form (29) can be discretized by Whitney edge elements, leading to a system of linear first-order differential equations in terms of the degrees of freedom of $\underline{a}$ (circulation of $\underline{a}$ on the edges of the FE mesh) [5].

In the approach proposed in this paper the surface-integral term in (29) is not exploited as such, but the two volume integrals considered for $\Omega_{m}$ will be reduced to surface integrals. Hereto we consider a local coordinate system $x y z$ on $\partial \Omega_{m}$, with the $x$-axis parallel to $\underline{n}$ and inward $\Omega_{m}$. Further ignoring the finite depth of $\Omega_{m}$ and the nonzero curvature of $\partial \Omega_{m}$, we write the magnetic vector potential in the transformed domain $\Omega_{m}$ as follows: $\underline{a}=\underline{a}_{t}(y, z) p(x, t)$, where $\underline{a}_{t}$ is tangential to $\partial \Omega_{m}$ and where $p(x, t)$ is differentiable with respect to $x$ $(0 \leq x<\infty)$. The two volume integrals on $\Omega_{m}$ are then worked out accordingly:

$$
\begin{aligned}
& \left(\nu \operatorname{curl} \underline{a}, \operatorname{curl} \underline{a}^{\prime}\right)_{\Omega_{m}}= \\
& \left\langle\operatorname{curl} \underline{a}_{t}, \operatorname{curl} \underline{a}_{t}^{\prime}\right\rangle_{\partial \Omega_{m}} \cdot \nu \int_{0}^{\infty} \partial_{x} p \partial_{x} p^{\prime} d x, \\
& \left(\sigma \partial_{t} \underline{a}, \underline{a}^{\prime}\right)_{\Omega_{m}}=\left\langle\partial_{t} \underline{a}_{t}, \underline{a}_{t}^{\prime}\right\rangle_{\partial \Omega_{m}} \cdot \sigma \int_{0}^{\infty} p p^{\prime} d x .
\end{aligned}
$$

Considering the $n$ pairs of basis functions $\alpha_{c k}(x)$ and $\alpha_{s k}(x)$ for the space discretisation of $p(t)$ and test functions $p^{\prime}(t)$, the integration along the $x$-axis in $(30,31)$ produces the elements of the matrices $[S]$ and $[T]$.

The number of spatial degrees of freedom of $\underline{a}$ on $\partial \Omega_{m}$ (and in $\Omega_{m}$ ) is thus equal to the number of edges on $\partial \Omega_{m}$ times $2 n$. In the 2-D case, adopting a one-component vector potential and nodal basis functions, the number of spatial degrees of freedom is equal to the number of nodes on the contour $\partial \Omega_{m}$ times $2 n$.

\section{Application eXAmple}

The 2-D application example concerns a conducting cylinder (circular cross-section with radius $R$ equal to $10 \mathrm{~cm}$; conductivity $\sigma$ equal to $610^{7} \mathrm{~S} / \mathrm{m}$ ) placed inside an inductor (coil sides of rectangular cross-section) in which the current is imposed. Only one quarter of the geometry is modeled. See Figure 2. All materials are non-magnetic $\left(\mu=\mu_{0}=\right.$ $\left.4 \pi 10^{-7} \mathrm{H} / \mathrm{m}\right)$. The extension of space unto infinity is considered by means of a transformation method.

Figure 2 shows the very fine discretisation of the cylinder near its surface, allowing precise calculations with very small $\delta / R$ ratios (without using the SIBC). Flux patterns with $\delta / R=0.5(f=1.69 \mathrm{~Hz})$ and $\delta / R=0.1(f=42.2 \mathrm{~Hz})$ are depicted in Figure 3. When applying the SIBC, only the mesh outside the cylinder is effectively considered.
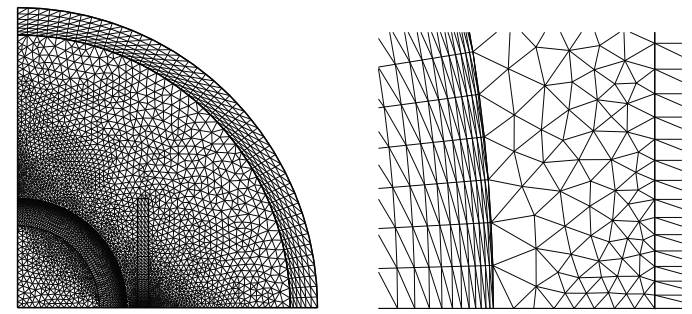

Fig. 2. FE model and mesh (1/4 of geometry) and detail of the FE mesh (the width of the outer layer of elements in the disk is equal to $0.3 \mathrm{~mm}$, i.e. $0.3 \%$ of the radius $R$ )
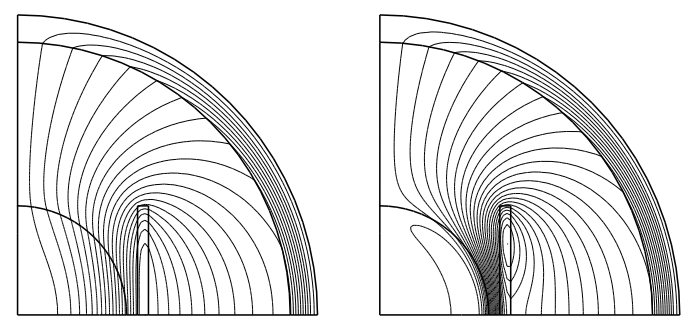

Fig. 3. Flux pattern (in phase with imposed sinusoidal current) with $\delta / R=$ 0.5 (left) and $\delta / R=0.1$ (right)

\section{A. Frequency-domain results}

The exact frequency-domain SIBC (28) has first been applied to our test case, with imposed sinusoidal current of unit amplitude and with $\delta / R$ ranging from $0.01(f=4.2 \mathrm{kHz})$ up to $1.5(f=0.19 \mathrm{~Hz})$ approximately. The accurate results obtained with the so-called fine model (without SIBC) serve as reference. Figure 4 shows the real and imaginary part of the flux linkage of the inductor (normalized with the flux at $0 \mathrm{~Hz}$ ). (Note that the imaginary part of the flux corresponds to the eddy-current losses in the cylinder, whereas its real part corresponds to the magnetic energy in the complete model.) We conclude from these results that the SIBC approach is accurate only if $\delta / R$ is smaller than 0.1 .

We next adopt the low-order approximation of the SIBC, with $f_{1}=1 \mathrm{kHz}\left(\delta_{1} / R=0.02\right)$ and further two discrete 

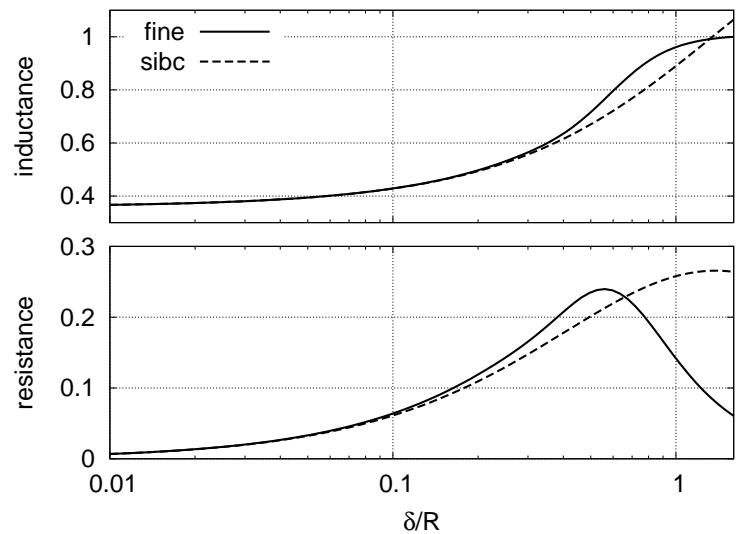

Fig. 4. Real and imaginary part of normalized flux linkage as a function of skin depth, calculated with fine model and with exact SIBC

frequencies being odd multiples of $f_{1}$, i.e. $f_{2} / f_{1}=3$ and $f_{3} / f_{1}=5\left(\right.$ or $\delta_{2} / \delta_{1}=1 / \sqrt{3}$ and $\delta_{3} / \delta_{1}=1 / \sqrt{5}$ ). Figure 5 shows the real and imaginary part of the normalized flux linkage obtained with $n$ equal to 1,2 and 3 .

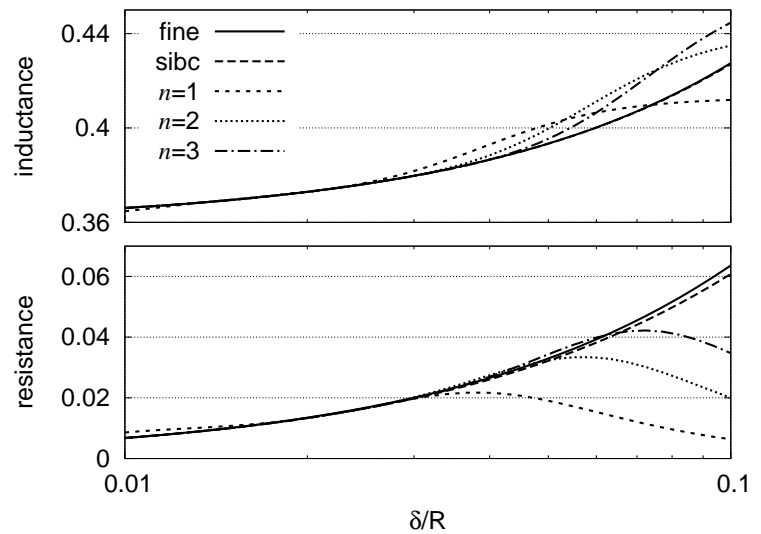

Fig. 5. Real and imaginary part of normalized flux linkage as a function of skin depth, calculated with fine model, exact SIBC and low-order SIBCs

\section{B. Time domain results}

A trapezoidal current varying between 1 and -1 at $1 \mathrm{kHz}$ is next considered. The current waveform together with the induction waveform in a point situated between the cylinder and the inductor are shown in Figure 6 for the first fundamental period $(1 \mathrm{~ms})$. For the low-order SIBCs, odd harmonics of $f_{1}=1 \mathrm{kHz}$ are considered, as above.

The magnetic energy and the joule losses in the cylinder have been calculated during the first period with the fine model (without SIBC) and the low-order SIBC ( $n$ equal to 1 and 2). A good convergence of the SIBC results towards the reference results is observed in Fig. 7.

\section{CONCLUSions}

The low-order SIBC approach presented in this paper is based on the resolution of the 1-D eddy-current problem in a semi-infinite slab considering a number of pairs of exponentially decreasing sine and cosine basis functions. The

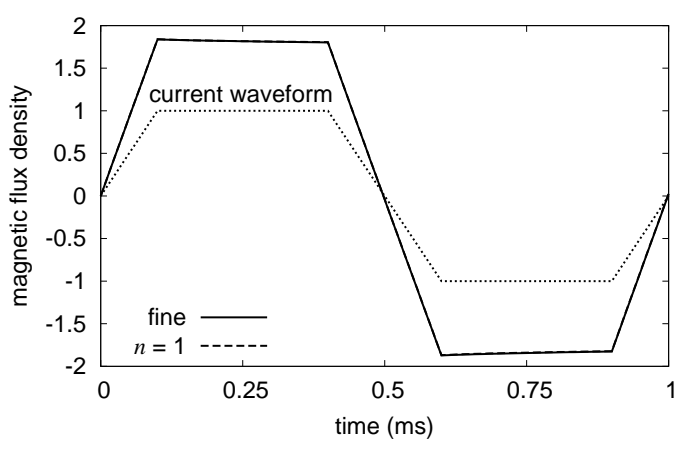

Fig. 6. Current waveform and normalized induction in a point versus time obtained with fine model and time-domain SIBC approach
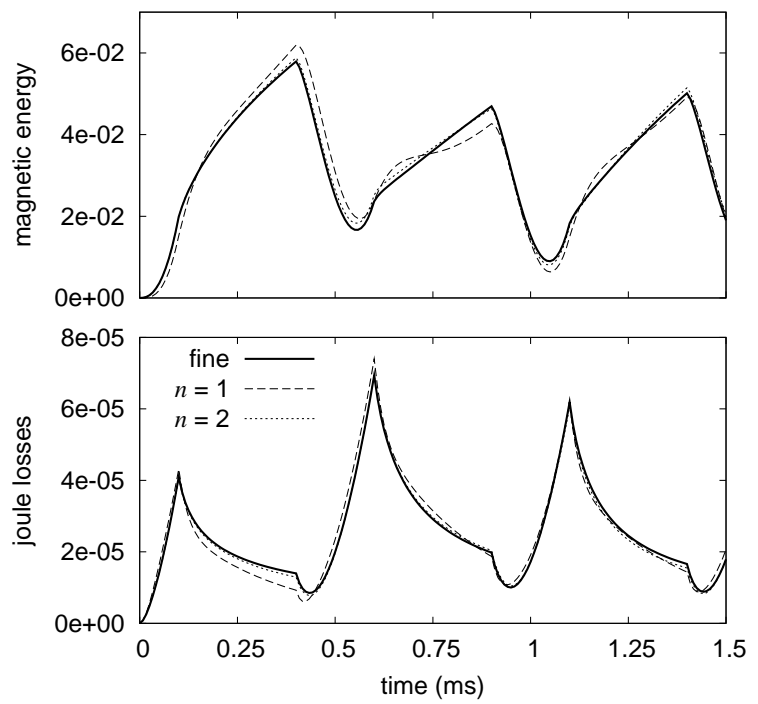

Fig. 7. Magnetic energy and joule losses in the cylinder versus time obtained with fine model and with time-domain approximation $(n=1$ and $n=2)$

choice of the associated discrete skin depths is application dependant but the stiffness and conductivity matrices to be evaluated for the application are essentially a function of the skin-depth ratios. The effectiveness of the SIBC approach has been clearly demonstrated with both frequency and time domain results.

\section{REFERENCES}

[1] S. Barmada, L. Di Rienzo, N. Ida and S. Yuferev, "The use of surface impedance boundary conditions in time domain problems: numerical and experimental validation," ACES Journal, vol. 19, pp. 76-83, July 2004.

[2] L. Krähenbühl, O. Fabrègue, S. Wanser, M. De Sousa Dias and A. Nicolas, "Surface Impedances, BIEM and FEM Coupled with 1D Non Linear Solutions to Solve 3D High Frequency Eddy Current Problems," IEEE Trans. Magn., vol. 33, pp. 1167-1172, March 1997.

[3] K.R. Davey and L. Turner, "Transient eddy current analysis for generalized structures using surface impedances and the fast Fourier transform," IEEE Trans. Magn., vol. 26, pp. 1164-1170, May 1990.

[4] D. Rodger and H.C. Lai, "A surface impedance method for 3D time transient problems," IEEE Trans. Magn., vol. 35, pp. 1369-1371, May 1999.

[5] A. Bossavit, "A rationale for edge-elements in 3-D fields computations," IEEE Trans. Magn., vol. 24, pp. 74-79, July 1988.

Manuscript received May 10, 2008. Corresponding author: J. Gyselinck (johan.gyselinck@ulb.ac.be). 